\title{
Role of MDCT Evaluation in Detection and Characterisation of Hepatic Mass Lesions
}

\author{
Dova Madhavi ${ }^{1}$ \\ ${ }^{1}$ Assistant Professor, Department of Radio Diagnosis \\ Corresponding author: Dova Madhavi, Assistant Professor, Department of Radio Diagnosis
}

DOI: http://dx.doi.org/10.21276/ijcmsr.2020.5.1.8

C) BY-NC-ND

How to cite this article: Dova Madhavi. Role of MDCT evaluation in detection and characterisation of hepatic mass lesions. International Journal of Contemporary Medicine Surgery and Radiology. 2020;5(1):A35-A38.

\section{A B S T R A C T}

Introduction: Multiphasic Computed Tomography (CT) has become the chief imaging modality for recognition and characterisation of focal liver lesions. CT has assumed primary role in evaluating hepatic masses. Study aimed to assess the imaging features of focal hepatic lesions on Multidetector Computed Tomography (MDCT) and its comparative assessment with histopathological results.

Material and Methods: This cross-sectional study was conducted in the Department of Radiodiagnosis in a tertiary care hospital for a period of 15 months. Adult patients of age group 20-60 years with focal hepatic lesions on abdominal imaging (USG), all patients presenting with deranged liver function or known cases of liver mass lesions were included in the study. All the values for the arterial phase, venous phase, portal phase and delayed phase were recorded and analysed along with the histopathological and biochemical analysis report. SPSS (Version 22.0) was used for analysis.

Results: As detected by MDCT, out of 90 focal liver lesions, benign focal liver lesions were 70(85\%) and malignant lesions were 12 (14.3\%). The diagnostic accuracy (efficiency) of MDCT was found to be $95 \%$ with predicted value (95\% Cl: 84.20 $96.75 \%)$. For the hepatocellular carcinoma cases, highly significant agreement $(p<0.001)$ was found between MDCT and biopsy techniques. The overall sensitivity, specificity, PPV, NPV and diagnostic accuracy for malignant lesions was found to be $83 \%, 97 \%, 83 \%, 97 \%$ and $95 \%$ respectively.

Conclusion: The study indicates MDCT to be highly sensitive in classifying the hepatic lesions into clinically relevant categories, making diagnosis and evaluation of lesion.

Keywords: Benign, Malignant, Hepatic Lesion, Contrast Enhanced Computed Tomography, Hepatic Cancer

\section{INTRODUCTION}

Focal liver lesions are best assessed and characterised by multiphasic CT. It is an effective aid in determining the number, location, and nature of such lesions and monitoring their size over period. In patients with cancer, the precise detection of metastatic disease at the time of diagnosis or during treatment remains crucial to organisation of the disease. Multidetector Computed Tomography (MDCT) represents an advance in CT technology that involves the use of a multiple-row detector array instead of the traditional single-row detector array used in spiral CT. This innovation allows scanning four to eight times faster than scanning with spiral CT. ${ }^{1}$ A triphasic spiral CT technique images the entire liver in arterial, portal, and equilibrium phases. ${ }^{2,3}$ Rapid availability and short scanning time made CT an ideal imaging modality. ${ }^{4,5}$ Inclusion of arterial phase imaging along with portal venous imaging improves the lesion detection especially in hyper vascular neoplasms. ${ }^{6}$ Most sensitive phase for lesion detection is portal phase whereas additional information on vascularity of the lesion is given by arterial and equilibrium phases. ${ }^{7}$ Multiple modalities in radiological imaging along with histopathological characteristics and clinical assessment are used to achieve a correct diagnosis.?
In the available literature there are fewer comprehensive studies about the character of multiphasic MDCT scan in characterising liver lesions under benign or malignant category and were conducted with a very small sample size. With the above background, this study was assumed as an effort to assess the role of MDCT in detection and characterisation of focal liver lesions with a larger sample size and help in deciding further course of management.

\section{MATERIAL AND METHODS}

This cross-sectional study was conducted in the Department of Radiodiagnosis in Tertiary care institute for a period of 15 months from January 2018 to April 2019 and total of 90 patients were included in the study. Sample size was calculated based on specification and Positive Predictive Value of $\mathrm{MDCT}^{8}$ using the formula:

$\mathrm{n}=\mathrm{Z} \alpha 2 \times \mathrm{P}(1-\mathrm{P}) / \mathrm{L}^{2}$

Sample Size $=22 \times 0.05 \times(1-0.05) /(0.05) 2=4 \times 0.05 \times 0.95$ $0.0025=76$

Where, $Z \alpha=1.96 \quad(95 \%$ CI), P=Prevalence $=5 \%$ (based on Tertiary Care Hospital) and L=Margin of error $=5 \%$ Loss $=10 \%=8$ Then sample size came out to be $=84 \sim 90$

Adult patients of age group 20-60 years with focal hepatic lesions on abdominal imaging (USG), all patients presenting 
with deranged liver function or known cases of liver mass lesions and the subjects who were willing to participate in the study and gave their written consent for the same were included in the study. Patients with traumatic liver lesions and patients with deranged blood clotting profile were excluded. After obtaining approval from the institutional ethical committee and written and informed consent from all the patients, they were subjected to a USG scan followed with Quadri-phasic CECT scan and biopsy. Obtained samples were sent for histopathology and biochemical analysis. All the values for the arterial phase, venous phase, portal phase and delayed phase were recorded, and all the patients were followed up till histopathological and biochemical report confirmation was done.

\section{STATISTICAL ANALYSIS}

Data so obtained were subjected to statistical analysis. Results were evaluated for the best modality through which benign and malignant lesions can be differentiated. Data analysis was done by SPSS software ${ }^{\circledR}$ version 22.0. Descriptive statistical analysis, which included frequency and percentages, was used to characterise the data. chi-square test was used for association between factors and $p<0.05$ was considered statistically significant.

\section{RESULT}

Out of 90 patients, maximum number of patients was from age group 30-39 years (33\%). The study was male preponderance (55\%). Another demographic details and lifestyle habits in which smoking, and alcohol consumption was done equally by $30 \%$ of patients. Mean age was found to be $38.72 \pm 11.55$ (20-60 year) (table- 1 ).

As per table 2 the most common complaint was found to

\begin{tabular}{|l|c|}
\hline Age (years) & $\mathbf{N ~ ( \% )}$ \\
\hline $20-29$ & $24(26)$ \\
\hline $30-39$ & $30(33)$ \\
\hline $40-49$ & $16(17)$ \\
\hline$>50$ & $20(22)$ \\
\hline Gender & $50(55)$ \\
\hline Male & $40(45)$ \\
\hline Female & $27(30)$ \\
\hline Addictions & $27(30)$ \\
\hline Smoking & $16(17)$ \\
\hline Alcohol & \\
\hline Tobacco & \\
\hline \multicolumn{2}{|c|}{ Table-1: Demographic details of the study participant } \\
\hline
\end{tabular}

\begin{tabular}{|l|c|}
\hline Complaints & $\mathbf{N}(\%)$ \\
\hline Acute abdomen & $61(68)$ \\
\hline Abdominal discomfort & $84(93)$ \\
\hline Palpable mass & $72(80)$ \\
\hline Abdominal mass & $42(47)$ \\
\hline Anorexia & $64(69)$ \\
\hline Fever & $68(71)$ \\
\hline Weight loss & $57(66)$ \\
\hline \multicolumn{2}{|c|}{ Table-2: Distribution of subjects according to complaints. } \\
\hline
\end{tabular}

\begin{tabular}{|l|l|c|}
\hline Lesions & Lesion category & N (\%) \\
\hline Abscess & Benign & $37(53)$ \\
\hline Cyst & Benign & $13(15)$ \\
\hline Haemangioma & Benign & $11(13)$ \\
\hline Hydatid cyst & Benign & $4(2)$ \\
\hline Hepatic granuloma & Benign & $5(4)$ \\
\hline Hepatocellular carcinoma & Malignant & $12(14)$ \\
\hline \multicolumn{2}{|c|}{ Table-3: Distribution of hepatic lesion based on MDCT }
\end{tabular}

\begin{tabular}{|l|c|c|}
\hline Results & N (\%) & Accuracy \\
\hline Matched & $90(95 \%)$ & $95 \%$ \\
\hline Unmatched & $10(5 \%)$ & \\
\hline \multicolumn{3}{|c|}{ Table-4: Diagnostic Accuracy of MDCT with Hepatic lesions } \\
\hline
\end{tabular}

\begin{tabular}{|l|c|c|c|}
\hline \multirow{2}{*}{ MDCT } & \multicolumn{2}{|c|}{ Biopsy } & \multirow{2}{*}{ Total } \\
\cline { 2 - 3 } & Benign & Malignant & \\
\cline { 2 - 3 } & $\mathbf{N}$ & $\mathbf{N}$ & \\
\hline Benign & $68(97 \%)$ & $2(3 \%)$ & 70 \\
\hline Malignant & $2(16 \%)$ & $10(84 \%)$ & 12 \\
\hline Total & $70(87 \%)$ & $12(14 \%)$ & 82 \\
\hline
\end{tabular}

Table-5: Validity and Reliability of MDCT with respect to Biopsy

be abdominal discomfort (93\%) followed by palpable mass (80\%). Anorexia and fever also seen while the least common complaint was found to be abdominal mass (47\%) (table-2). As per table 3 Out of 90 , benign focal liver lesions were 70 (87\%) and malignant lesions were 12 (14\%). Among the benign lesions, maximum cases were of abscess (53\%) Others were Cyst (15\%), Haemangioma (13\%), Hydatid cyst (2\%) and Hepatic Granuloma (4\%). Among the malignant lesions MDCT finding was of Hepatocellular carcinoma (14\%) (table-3).

as per table 4 For detecting various types of lesions, the results of MDCT matched with biopsy in 90\% cases while in 10\% cases the results didn't match. So, the diagnostic accuracy (efficiency) of MDCT was found to be $90.5 \%$ with predicted value (95\% CI: $84.20-96.75 \%)$

To determine the validity and reliability MDCT with respect to biopsy shows benign cases by biopsy and among the malignant cases detected byMDCT only $84 \%$ cases confirmed as malignant by biopsy. Highly significant association (chi$\mathrm{sq}=44.51, \mathrm{p}<0.001$ ) was found between MDCT and biopsy techniques. The overall sensitivity, specificity, PPV, NPV and diagnostic accuracy for malignant lesions were found to be $83 \%, 97 \%, 83 \%, 97 \%$ and $95 \%$ respectively.

\section{DISCUSSION}

In the available literature there are few Indian comprehensive studies about the role of Multiphasic MDCT scan in improving the diagnostic accuracy of characterising the malignant liver lesions. Risk factors and complaints of the study subjects: Habits like smoking, alcohol consumption and tobacco chewing that are considered as risk factors for malignant liver lesions were present in majority of subjects in our study. Such habits were also proven by Simonetti RG et al., to be risk factors for malignant liver lesions. ${ }^{8}$ Validity and reliability for detecting malignant liver lesions: In present 
study, the overall sensitivity and specificity of MDCT with respect to biopsy, for establishing their validity and reliability in detecting malignancy in liver lesions, was found to be $83 \%$ and $97 \%$, respectively. In addition, in a meta-analysis of hepatic metastases from cancers of the gastrointestinal tract, Kinkel K et al., reported a mean sensitivity of $72 \%$ for CT, based on 25 publications that included 1747 patients. ${ }^{9}$ In a study by Khalid $\mathrm{M}$ et al., the diagnostic accuracy of metastatic focal hepatic lesions by MRI was $55 \%$ as compared with $17 \%$ for $\mathrm{CT}^{10}{ }^{10}$ In this study, more recent comparisons of noninvasive imaging modalities, primarily MDCT and USG, have shown equally accurate if not better lesion detection by MDCT. However, in the present study, cases of abscess show highly significant agreement between MDCT and biopsy techniques. The overall sensitivity and specificity of MDCT was found to be $99.3 \%$ and $97.1 \%$ respectively. For the cases of cyst, highly significant agreement was found between MDCT and biopsy techniques. The overall sensitivity, specificity, PPV, NPV and diagnostic accuracy of MDCT was found to be $97.9 \%, 96.6 \%, 87.9 \%, 97.9 \%$ and $96.7 \%$ respectively. Ahirwar $\mathrm{CP}$ et al., added that with sensitivity of $91.3 \%$, specificity $97.8 \%$, PPV $91.3 \%$ and NPV $97.8 \%$ ( $\mathrm{p}$-value $<0.001$, kappa value 0.847 ), Triple phase CT is excellent diagnostic modality for characterisation and better evaluation of hepatic masses. ${ }^{11}$ The hydatid cyst and hepatocellular carcinoma cases showed overall sensitivity and specificity of $97.4 \%, 96.1 \%$, and $96.9 \%, 90.4 \%$, respectively. In contrast Hassan and their colleagues had sensitivity and specificity of haemangiomas to be $76.9 \%, 88.4 \%$ and for MRI $85.2 \%$ and $91.2 \%$, whereas, in hepatocellular carcinoma, the sensitivity and specificity of CT were $62 \%$ and $83.3 \%$ and for MRI were $90.3 \%$ and $87.5 \%$ in metastases, sensitivity and specificity of CT were $60 \%$ and $84 \%$ and for MRI were $76.2 \%$ and $87.8 \% .{ }^{12}$ Eighty percent of the false-positive MRI results and $67 \%$ of the false-positive CT results were primarily attributed to arterio-portal shunt. Furthermore, cirrhosis-related benign nodules may exhibit predominant hypo-attenuation on contrast-enhanced portal or delayed phase $\mathrm{CT}$ image ${ }^{13}$ and might not be differentiated from hypo-vascular HCC. In another study 33\%, of falsepositive findings on MDCT were attributed to prominent cirrhosis-related nodules. In a study by Matilde NM et al., $92 \%$ of the 100 lesions demonstrate arterial enhancement. ${ }^{14}$ When all lesions with circumferential ring enhancement were considered, malignancy was predicted with $86.8 \%$ specificity for metastasis. As found in this study and others, benign and malignant lesions may overlap in appearances, for example, homogeneous pattern can be exhibited by $\mathrm{HCC}$, haemangiomas and $\mathrm{FNH} .{ }^{14}$ In addition, according to Pattanayak SK et al., the conspicuity of a liver lesion depends on the attenuation difference between the lesion and the normal liver. ${ }^{15}$ Due to low contrast in non-enhanced $\mathrm{CT}$ scans between tumor tissue and surrounding liver parenchyma, tumors become invisible. Usually, a combination of different features like enhancement pattern, pathological features like presence of fat, blood, calcifications, cystic or fibrotic component in the liver lesions along with clinical history is used to frame the differentials. Gupta K et al., also added a significant finding about the role of $\mathrm{CT}$ in evaluation of parenchymal focal lesions of liver and concluded that MDCT is a highly sensitive non-invasive tool for detection and characterisation of focal hepatic lesions. ${ }^{16}$

\section{CONCLUSION}

The results of this study demonstrate MDCT to be highly sensitive in categorisation the hepatic lesions into clinically applicable categories which helps in achieving correct diagnosis and evaluation of lesion. This study opens new likelihoods of prevention of liver disease with early detection and resulting management of hepatic lesions.

\section{REFERENCES}

1. Berland LL, Smith JK. Multi-detector array CT: once again, technology creates new opportunities (editorial). Radiology. 1998;20(9):327-29.

2. Bonaldi VM, Bret PM, Reinhold C, Atri M. Helical computed tomogram of liver, value of an early hepatic arterial phase. Radiology. 1995;19(7):357-63.

3. Francis IR, Cohan RH, McNulty NJ, Platt JF, Korobkin M, Gebremariam A, et al. Multi-detector CT of the liver and hepatic neoplasms: Effect of multiphasic imaging on tumor conspicuity and vascular enhancement. AJR Am J Roentgenol. 2003;18(1):1217-24.

4. Ichikawa T, Saito K, Yoshioka N, Tanimoto A, Gokan $\mathrm{T}$, Takehara $\mathrm{Y}$, et al. Detection and characterization of focal liver lesions: A Japanese phase III, multicentre comparison between gadoxetic acid disodium enhanced magnetic resonance imaging and contrast enhanced computed tomography predominantly in patients with hepatocellular carcinoma and chronic liver disease. Invest Radiol. 2010;4(5):133-41.

5. Hammersting R, Huppertz A, Breuer J, Balzer T, Blakeborough A, Carter R, et al. Diagnostic efficacy of gadoxetic acid (Primovist)-enhanced MRI and spiral CT for a therapeutic strategy: Comparison with Intraoperative and histopathologic findings in focal liver lesions. Eur Radiol. 2008;1(8):457-67.

6. Soyer P, Sirol M, Fargeaudou Y, Duchat F, Hamzi L, Boudiaf M, et al. Differentiation between true focal liver lesions and pseudolesions in patients with fatty liver: evaluation of helical CT criteria. Eur Radiol. 2010;20(1):1726-37.

7. Van Leeuven MS, NoordzijJ, Feldberg MA, Hennipman AH, Doorneewaard [7] H. Focal Liver lesions; characterization with tri-phasic computed tomography. Radiology. 1996;20(1):327-36.

8. Simonetti RG, Cammà C, Fiorello F, Politi F, D'Amico G, Pagliaro L. Hepatocellular carcinoma. A worldwide problem and the major risk factors. Dig Dis Sci. 1991;36(1):962-72.

9. Kinkel K, Lu YM. Both Detection of hepatic metastases from cancers of the gastrointestinal tract by using noninvasive imaging methods (US, CT, MR imaging, PET): a meta-analysis. Radiology. 2002;224(5):748-56.

10. Khalid M, John R, Cheistine O.MRI characterization of $124 \mathrm{CT}$ indeterminate focal hepatic lesions: Evaluation of clinical utility HPB (Oxford). 2007;9(3):102-08.

11. Ahirwar CP, Patil A, Soni N. Role of triple phase computed tomography findings for evaluation of hepatic lesions. Int J Res Med Sci. 2016;4(1):3576-83. 
12. Hassan A, Refaa A, Khaledah D, Mahmoud A. Sixtyfour multi-slice computed tomography and magnetic resonance imaging in evaluation of hepatic focal lesions. The Egyptian Journal of Radiology and Nuclear Medicine. 2011;4(2):101-10.

13. Kim SH, Choi D, Kim SH. Ferucarbotran enhanced MRI versus triple-phase MDCT for the preoperative detection of hepatocellular carcinoma. AJR. 2005;184(2):1069-76.

14. Matilde NM, Olcott Eric W, Brooke Jeffrey R, Lamm Robert L, Beaulieu Christopher F, Jain Kiran A. et al.Focal liver lesions: pattern-based classification scheme for enhancement at arterial phase CT. Radiology. 2000;215(3):746-51.

15. Pattanayak SK, Bahinipati P, Mohapatra SSG. Triphasic multidetector computed tomography in detection and characterisation of solitary liver lesions. Indian Journal of Research. 2018;7(7):44-46.

16. Gupta K, Gauba N, Gupta G. Role of computed tomography in evaluation of parenchymal focal lesions of liver. Journal of Evolution of Medical and Dental Sciences. 2015;4(36):6257-68

Source of Support: Nil; Conflict of Interest: None

Submitted: 11-12-2019; Accepted: 26-12-2019; Published online: 24-01-2020 